\title{
Local Constructive Set Theory and Inductive Definitions
}

Peter Aczel

\section{Introduction}

Local Constructive Set Theory (LCST) is intended to be a local version of constructive set theory (CST). Constructive Set Theory is an open-ended set theoretical setting for constructive mathematics that is not committed to any particular brand of constructive mathematics and, by avoiding any built-in choice principles, is also acceptable in topos mathematics, the mathematics that can be carried out in an arbitrary topos with a natural numbers object. We refer the reader to [2] for any details, not explained in this paper, concerning CST and the specific CST axiom systems $\mathbf{C Z F}$ and $\mathbf{C Z F}^{+} \equiv \mathbf{C Z F}+\mathbf{R E A}$.

CST provides a global set theoretical setting in the sense that there is a single universe of all the mathematical objects that are in the range of the variables. By contrast a local set theory avoids the use of any global universe but instead is formulated in a many-sorted language that has various forms of sort including, for each sort $\alpha$ a power-sort $\mathscr{P} \alpha$, the sort of all sets of elements of sort $\alpha$. For each sort $\alpha$ there is a binary infix relation $\in_{\alpha}$ that takes two arguments, the first of sort $\alpha$ and the second of sort $\mathscr{P} \alpha$. For each formula $\phi$ and each variable $x$ of sort $\alpha$, there is a comprehension term $\{x: \alpha \mid \phi\}$ of sort $\mathscr{P} \alpha$ for which the following scheme holds.

Comprehension: $(\forall y: \alpha)[y \in \alpha\{x: \alpha \mid \phi\} \leftrightarrow \phi[y / x]]$.

Here we use the notation $\phi[a / x]$ for the result of substituting a term $a$ for free occurences of the variable $x$ in the formula $\phi$, relabelling bound variables in the standard way to avoid variable clashes.

Our use of the terminology local for a version of a set theory has its origin in the use of that term by John Bell in his book [5]. His notion of a local set theory is a

Peter Aczel

Schools of Computer Science and Mathematics, University of Manchester,e-mail: petera@cs.man.ac.uk 
certain kind of syntactic version of the category theoretic notion of a topos. Each of his local set theories uses a local language that has type symbols built up from ground type symbols. The type symbols have various forms including the form of a power type PA, where $\mathbf{A}$ is a type. There are terms of each type and the set-like terms of the local language are the terms of some power type. So, in a local set theory, there is no global universe of sets, but each set has to be understood as local to some power type. Here we will keep to this general idea but will not be using the precise details of the formulations in Bell's book. In particular we prefer to use the word sort rather than type. Our first example of a local set theory will be what we will call Local Intuitionistic Zermelo (LIZ). This is essentially a variant of what Bell has called at the end of chapter 7 of [5], the free naturalised local set theory; i.e. the local set theory for the free topos with a natural numbers object. It is also natural to describe it as a version of intuitionistic higher order arithmetic.

There are several reasons for our interest in the setting up of a local version of CST. One reason is in connection with the formulation of predicative and generalised predicative versions of the notion of an elementary topos. We expect that a local (generalised) predicative axiom system for CST will have as its category theoretic models categories that are (generalised) predicative toposes, according to some suitable weakening of the notion of an elementary topos. Some category theorists dislike global set theories because they claim that the focus of global theories on the structure of the binary membership relation on the universe of sets is irrelevent to mainstream mathematics. So a local approach to CST may be more appealing to a category theorist interested in constructive mathematics and the carrying over of the beautiful, but fully impredicative apparatus, of topos theory to the generalised predicative context.

Another reason for our interest in the development of a local version of CST is to do with the dependent type theoretical setting for constructive mathematics initiated by Per Martin-Löf. That setting aims to provide a philosophically motivated foundational framework for constructive mathematics that makes explicit the fundamental notions. It is the natural translation of the CST axiom systems such as $\mathbf{C Z F}$ and $\mathbf{C Z F}^{+}$into formulations of the type theoretic setting that have been used to justify the claim that those axiom systems are constructively acceptable. Although the translation is indeed natural it is technically somewhat complicated due to the transfinitely iterative nature of the global universe. Some of that complication can be avoided when directly interpreting the language of local set theory into type theory. We consider this important in connection with our third reason for our interest in a local version of CST.

In recent years there has been a growing interest in the development of old and new areas of constructive mathematics and there have been competing settings for this such as the Bishop style approach and the type theoretical, set theoretical and category theoretical approaches. Each has its advantages. The Bishop style approach is informal and works directly with the intensional constructive notions. The type theoretical approach is a more formal philosophically motivated approach. The set theoretical approach is fully extensional and is close to the mainstream set theoretical approach to classical mathematics. The category theoretic approach is more 
conceptual with its focus on the algebraic structure of the fundamental mathematical notions. Definitions and results formulated in one approach should carry over to the other approaches. But this is not always a straightforward matter.

For example let us focus on the relationship between type theoretical and set theoretical constructive mathematics. In some presentations of work in constructive mathematics definitions and results are given in an ambiguous style, intended to be understood in both the type theoretical and the set theoretical setting. There is a danger that such a style leads to a lack of rigour. So we advocate another approach. Develop constructive mathematics so that it can be straightforwardly formalised in suitable axiom systems for local CST. As local CST has a straightforward interpretation in global CST and a fairly straightforward direct type theoretic interpretation we get a simple rigorous approach to having definitions and results simultaneously in both settings.

The details of the direct type theoretic interpretation requires more type-theoretic treatment than seems appropriate for this paper and so has been left for another occasion. Suffice it to state here that each sort $\alpha$ is interpreted as a setoid $[[\alpha]]$; i.e.

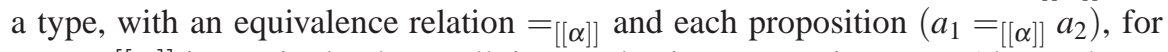
$a_{1}, a_{2}:[[\alpha]]$ is required to be small; i.e. a value in a type universe $U$. Also each setterm of sort $\mathscr{P} \alpha$ will be interpreted as an object pair $(A, f):(\Sigma X: U)(X \rightarrow[[\alpha]])$.

We will want our local version of CST to have a straightforward interpretation in global CST. But some care is needed in setting up the language. Classical set theory has the powerset axiom and the full separation scheme. So an interpretation of a local set theory in classical set theory has each sort $\alpha$ interpreted as a set $[[\alpha]]$ with the sort $\mathscr{P} \alpha$ interpreted as the powerset Pow $([[\alpha]])$ and each comprehension term $\{x: \alpha \mid \phi\}$ of sort $\mathscr{P} \alpha$ interpreted as a subset of $[[\alpha]]$.

But a key feature of CST is that the powerset axiom and the full separation scheme are not available, as these are too impredicative. So, instead of interpreting each sort as a set, the interpretation of our local version of CST will interpret each sort as a class, with $[[\mathscr{P} \alpha]]$ interpreted as the powerclass of $[[\alpha]]$; i.e. the class Pow $([[\alpha]])$ of all subsets of the class $[[\alpha]]$. Also, each comprehension term $\{x: \alpha \mid \phi\}$ of sort $\mathscr{P} \alpha$ will now be interpreted as a subclass of the class $[[\alpha]]$ which, in general, may not be a set in $[[\mathscr{P} \alpha]]$.

Class terminology and notation provides a useful device when working in classical axiomatic set theory. It proves to be even more useful when working in CST when many comprehension terms that represent sets in classical set theory can only be taken to be classes in CST. To treat classes in a set theory in a flexible way, without making them values in the range of bound variables, it is convenient to formulate a set theory in a suitable free logic. In general, a free logic allows the use of terms which may not represent values in the range of the variables.

There are a variety of approaches to the setting up of a free logic. For example some approaches such as those of Beeson, [4], are intended for use with function symbols that may be interpreted as partial functions, so that terms may be undefined in an interpretation. In such an approach it is natural to require equality and other relation symbols to be strict in the sense that they are only intended to hold for arguments that are in the range of the bound variables. In our approach to free logic 
we will be more liberal. We do not want equality to be strict, as equality between classes has a natural extensional treatment. Also the membership relation should only be strict in its first argument.

A key axiom scheme of $\mathbf{C Z F}$ is the set induction scheme. It is a suitably constructive version of the classical foundation axiom that expresses that all sets are well-founded. More specifically the scheme states that the universe is inductively generated as the smallest class such that every subset of the class is an element of the class. By making essential use of the scheme we have a class induction metatheorem for CZF. The metatheorem expresses that a general kind of inductive definition can be used to inductively generate a class as the smallest class satisfying closure conditions specified by the inductive definition. Moreover, by making essential use of the axiom REA of $\mathbf{C Z F}^{+}$, for certain inductive definitions the inductively generated class will be a set. In addition, using REA again, a useful set-compactness result concerning set inductive definitions can be obtained.

These inductive definition results can play an important role in the development of constructive mathematics in CST. See, for example [1], where it is shown using set compactness that every inductively generated formal topology is set-presentable. So we would like to have these inductive definition results available in our local CST. But there is a problem with the proofs of these results in local CST. The results can be formulated in local CST. But the proofs of the results use the set induction scheme and the axiom REA, a scheme and axiom that are global and do not have direct local formulations. The other axioms and schemes of $\mathbf{C Z F}$ and $\mathbf{C Z F}^{+}$do have local formulations. So we will introduce new axiom systems $\mathbf{C Z F I}$ and $\mathbf{C Z F}{ }^{*}$ that have axioms and schemes that directly express the inductive definition results of $\mathbf{C Z F}$ and $\mathbf{C Z F}^{+}$respectively. They are both extensions of the axiom system $\mathbf{C Z F}^{-}$ obtained from a formulation of CZF by leaving out the set induction scheme. We will see that both $\mathbf{C Z F I}$ and $\mathbf{C Z F}{ }^{*}$ have local versions

We review the CST axiom systems $\mathbf{C Z F}, \mathbf{C Z F}^{+}$and $\mathbf{C Z F}^{-}$in section 2. We also discuss the inductive definition results that can be proved and formulate the axiom systems CZFI and $\mathbf{C Z F}^{*}$. In section 3 we introduce our free logic and the free versions of the CST axiom systems. We go on, in section 5, to formulate our local versions of these axiom systems. But before that we introduce the ideas of local set theory by formulating a local version of intuitionistic Zermelo set theory. We give an application of inductive definitions to well-founded trees in section 6. 


\section{Inductive Definitions in CST}

In this section we review results concerning inductive definitions that have been obtained in the CST axiom systems $\mathbf{C Z F}$ and $\mathbf{C Z F}{ }^{+}$.

\subsection{Inductive Definitions in CZF}

The axiom system CZF is formulated in the usual first order language of axiomatic set theory with equality and membership as the two relation symbols. It has the axioms and rules of inference for intuitionistic predicate logic with equality and uses the non-logical axioms and schemes of Extensionality, Pairing, Union, Infinity, Restricted Separation, Strong Collection, Subset Collection and Set Induction. See [2] for the details of these axioms and schemes. Alternatively the reader may get a good enough idea by looking at the presentation of the axiom system $\mathbf{C Z F}_{\mathbf{f}}$ in the next section. Here we just consider the Infinity axiom, which states that there is an $\omega$-inductive set, where we define a class $A$ to be $\omega$-inductive if $\emptyset \in A$ and $(\forall x \in A)[x \cup\{x\} \in A]$.

Let $\mathbf{C Z F}^{-}$be obtained from $\mathbf{C Z F}$ first, by leaving out from the axioms and schemes the set induction scheme, and second by strengthening the axiom of Infinity to the axiom of Strong Infinity and adding the Mathematical Induction Scheme. Strong Infinity states that there is a smallest $\omega$-inductive set, while Mathematical Induction states that the smallest $\omega$-inductive set is a subset of each $\omega$-inductive class. Note that both Strong Infinity and Mathematical Induction can be derived in CZF.

The axiom system $\mathbf{C Z F}{ }^{-}$is fully predicative. It is the set induction scheme that gives $\mathbf{C Z F}$ its logical strength. That scheme expresses that the universe of sets is the smallest class such that every subset of the class is an element of the class. Although the scheme is not predicative in the traditional sense it is not fully impredicative either, as it does not imply the powerset axiom or the full separation scheme. It is natural to call it generalised predicative, as it is predicative relative to certain kinds of inductive definition which may be infinitary rather than the finitary inductive definitions which are acceptable in predicative mathematics.

It will not be difficult to formulate a local version of $\mathbf{C Z F}^{-}$. But the set induction scheme is a global property of the universe of sets and there seems to be no direct way to formulate a local version of that scheme so as to obtain a local version of the whole of $\mathbf{C Z F}$. An important metatheorem about $\mathbf{C Z F}$, which would seem to express the logical strength of $\mathbf{C Z F}$, states that class inductive definitions of classes hold for CZF. We shall see, in section 5, that the metatheorem can be formulated and derived for an extension of the local version of $\mathbf{C Z F}^{-}$and we will take that extension as our local version of CZF. The additional axioms of the extension will directly express that set inductive definitions of classes hold. But in this section we consider inductive definitions in the global context. 
We think of an inductive definition as an abstract axiom system having (inference) steps $X / a$ consisting of a (possibly infinite) set $X$ of premisses and a conclusion $a$. The theorems of the axiom system form the smallest class closed under the inference steps; i.e. for each step $X / y$, if the premisses are in the class then so is the conclusion. Any class $\Phi$ can be viewed as a class inductive definition whose steps $X / a$ are the ordered pairs $(X, a)$ in $\Phi$. A class is defined to be $\Phi$-closed if, for each step $X / a$ of $\Phi$, if every element of $X$ is in the class then so is $a$. The class inductively defined by $\Phi$, if it exists, is the smallest $\Phi$-closed class.

Definition: 2.1 A set theoretical axiom system $T$ has the class induction property if, for each class $\Phi$ of $T$ there is a smallest $\Phi$-closed class $I(\Phi)$ of $T$; i.e. there is a class I such that the following are derivable in $T$.

1. I is $\Phi$-closed, and

2. if the class $A$ is $\Phi$-closed then $I \subseteq A$.

The metatheorem may now be formulated as follows.

Theorem: 2.2 (Class Induction Metatheorem for CZF) The theory CZF has the class induction property.

Note that the Set Induction Scheme may be restated as $V=I(\Phi)$, where $\Phi$ is the class of all pairs $(X, X)$. The scheme is clearly a global property about the universe $V$.

It will be straightforward to formulate a local version of the predicative system $\mathbf{C Z F}^{-}$. We now formulate an extension $\mathbf{C Z F I}$ of $\mathbf{C Z F}^{-}$by adding a new binary infix relation symbol $\vdash$ to the language satisfying the following axioms and scheme where, for each class $\Phi$,

$$
I(\Phi)=\left\{x \mid \Phi_{0} \vdash x \text { for some subset } \Phi_{0} \text { of } \Phi\right\} .
$$

ind0: $\vdash$ is monotone in its first argument; i.e. for all sets $\Phi, \Phi^{\prime}$,

$$
\Phi \subseteq \Phi^{\prime} \Rightarrow(\forall x)\left[\Phi \vdash x \Rightarrow \Phi^{\prime} \vdash x\right] .
$$

ind1: For all sets $\Phi$ the class $I(\Phi)$ is $\Phi$-closed.

ind2: For each class $A$, if $\Phi$ is any set such that $A$ is $\Phi$-closed then $I(\Phi) \subseteq A$.

Note that, because of ind0, $I(\Phi)=\{x \mid \Phi \vdash x\}$ for each set $\Phi$. Also ind1 and ind2 combine to state that for each set $\Phi$, the class $I(\Phi)$ is the smallest $\Phi$-closed class. The next result states that, for CZFI, $I(\Phi)$ is the smallest $\Phi$-closed class, even when $\Phi$ is a class that may not be a set.

Theorem: 2.3 (Class Induction Metatheorem for CZFI) The axiom system CZFI has the class induction property.

Proof. Let $\Phi$ be a class. We first show that $I(\Phi)$ is $\Phi$-closed. So let $X / a$ be a step of $\Phi$ such that $X \subseteq I(\Phi)$. We must show that $a \in I(\Phi)$. By our assumption,

$$
(\forall x \in X)\left(\exists \Phi_{0} \in \operatorname{Pow}(\Phi)\right) \Phi_{0} \vdash x .
$$


So, by Strong Collection, there is a subset $\mathscr{Y}$ of $\operatorname{Pow}(\Phi)$ such that

$$
(\forall x \in X)\left(\exists \Phi_{0} \in \mathscr{Y}\right) \Phi_{0} \vdash x
$$

Now let $\Phi_{1}=\{(X, a)\} \cup \cup \mathscr{Y}$ and observe that $\Phi_{1}$ is a subset of $\Phi$ having $X / a$ as a step, with $X \subseteq I\left(\Phi_{1}\right)$ so that, by ind1, $a \in I\left(\Phi_{1}\right) \subseteq I(\Phi)$ as the operator $I$ is monotone.

It remains to show that $I(\Phi)$ is a subclass of each $\Phi$-closed class $A$. So let $a \in I(\Phi)$; i.e. $\Phi_{0} \vdash a$ for some subset $\Phi_{0}$ of $\Phi$. If $A$ is $\Phi$-closed then it is $\Phi_{0}$-closed so that, by ind2, $a \in I\left(\Phi_{0}\right) \subseteq A$, as desired.

\subsection{Inductive Definitions in $\mathrm{CZF}^{+}$}

A useful strengthening of $\mathbf{C Z F}$ is the axiom system $\mathbf{C Z F}^{+} \equiv \mathbf{C Z F}+\mathbf{R E A}$. Here REA is the regular extension axiom, which states that every set is a subset of a regular set. A regular set is an inhabited transitive set ${ }^{1} A$, such that for each $a \in A$, if $R \subseteq a \times A$ such that $(\forall x \in a)(\exists y \in A)(x, y) \in R$ then there is $b \in A$ such that both $(\forall x \in a)(\exists y \in b)(x, y) \in R$ and $(\forall y \in b)(\exists x \in a)(x, y) \in R$. The following results about inductive definitions may be derived in $\mathbf{C Z F} \mathbf{F}^{+}$, see [2, 3].

A set $B$ is a set bound for a class $\Phi$ if, for each step $Y / z$ of $\Phi$, there is $b \in B$ and a surjective $f: b \rightarrow Y$. The class $\Phi$ is defined to be bounded if it has a set bound and for each set $Y$ the class $\{z \mid(Y, z) \in \Phi\}$ is a set. Note that, in $\mathbf{C Z F}$, each set is bounded. For each set $\Phi_{0}$ let $I_{0}\left(\Phi_{0}\right)$ be the intersection of all $\Phi_{0}$-closed sets; i.e. the class $\bigcap\left\{Y \mid Y\right.$ is a $\Phi_{0}$-closed set $\}$. Also, for each class $\Phi$ let

$$
I(\Phi)=\bigcup\left\{I_{0}\left(\Phi_{0}\right) \mid \Phi_{0} \in \operatorname{Pow}(\Phi)\right\}
$$

Bounded Induction Scheme (BIS): For each class $\Phi$, the class $I(\Phi)$ is a subclass of each $\Phi$-closed class and hence is the smallest $\Phi$-closed class. Moreover if $\Phi$ is bounded then $I(\Phi)$ is a set and so $I(\Phi)=I_{0}(\Phi)$.

Another useful result of $\mathbf{C Z F}{ }^{+}$is the Set Compactness property for set inductive definitions. See [2] for the original result and [3] for a proof of the more recent improvement, SSC.

Strong Set Compactness Property (SSC): For every set $\Phi$ there is a set $B$ of subsets of $\Phi$ such that for every subset $\Phi^{\prime}$ of $\Phi$ every element of $I_{0}\left(\Phi^{\prime}\right)$ is in $I_{0}\left(\Phi_{0}\right)$ for some subset $\Phi_{0}$ of $\Phi^{\prime}$ that is in $B$.

Theorem: $2.4\left(\mathbf{C Z F}^{+}\right)$Each instance of $\mathbf{B I S}$ can be derived as can the statement SSC.

\footnotetext{
${ }^{1}$ i.e. a set that has an element and is such that it is a subset of its powerset.
} 
Let $\mathbf{C Z F}^{*} \equiv \mathbf{C Z F}+\mathbf{B I S}+\mathbf{S S C}$. As each set is bounded we get the following consequence of BIS.

Corollary: $2.5\left(\mathbf{C Z F}^{*}\right)$ For each set $\Phi$ the class $I_{0}(\Phi)$ is a set and so $I_{0}(\Phi)=$ $I(\Phi)$, the smallest $\Phi$-closed class.

For classes $\Phi$ and $A$ let $I(\Phi, A)=I\left(\Phi_{A}\right)$, where $\left.\Phi_{A}=\Phi \cup\{(\emptyset, x) \mid x \in A\}\right)$. Note that $I(\Phi, A)$ is the smallest $\Phi$-closed class that includes $A$. It immediately follows from corollary 2.5 that if $\Phi$ is a set then $I(\Phi, A)$ is a set for each set $A$. We have the following consequence of SSC.

Corollary: 2.6 (Set Compactness for $\mathbf{C Z F}^{*}$ ) For all sets $\Phi, A$ there is a set $B$ of subsets of $A$ such that, for all sets $A^{\prime} \subseteq A$, each element of $I\left(\Phi, A^{\prime}\right)$ is an element of $I\left(\Phi, A_{0}\right)$ for some subset $A_{0}$ of $A^{\prime}$ that is in $B$.

\section{The Free Version of CST}

\subsection{A Free Logic}

We present our free version of intuitionistic predicate logic with equality. We assume that formulae are generated from the atomic formulae in the usual way using the logical constants $\perp$ and $T$, the binary connectives $\wedge, \vee$ and $\rightarrow$ and the quantifiers $(\forall x)$ and $(\exists x)$ for each variable $x$. Abbreviations for $\neg$ and $\leftrightarrow$ are defined as usual.

We first consider the following Hilbert style axiomatisation of Intuitionistic logic with equality and then present our free modification. A standard set of axioms together with the rule of modus ponens will do for intuitionistic propositional logic. We just focus on the axioms and rules for equality and the quantifiers. The equality axioms are as follows, where $a, a_{1}, a_{2}$ are terms and $x$ is a variable.

$$
a=a \quad\left(a_{1}=a_{2}\right) \wedge \phi\left[a_{1} / x\right] \rightarrow \phi\left[a_{2} / x\right]
$$

We consider the following standard quantifier rules and axioms, where $x$ is a variable not free in the formula $\theta$ and $a$ is any term.

$$
\begin{array}{ll}
\frac{\theta \rightarrow \phi}{\theta \rightarrow(\forall x) \phi} & (\forall x) \phi \rightarrow \phi[a / x] \\
\phi[a / x] \rightarrow(\exists x) \phi & \frac{\phi \rightarrow \theta}{(\exists x) \phi \rightarrow \theta}
\end{array}
$$

We use $a \downarrow$ to abbreviate $(\exists x)[a=x]$, where the variable $x$ is chosen not to occur free in the term $a$. This expresses that the term $a$ is in the range of the variables. For our free logic we modify the two quantifier axioms as follows.

$$
a \downarrow \wedge(\forall x) \phi \rightarrow \phi[a / x] \quad a \downarrow \wedge \phi[a / x] \rightarrow(\exists x) \phi
$$

We also need the axiom, $y \downarrow$, for each variable $y$. 


\subsection{The Axiom System $\mathrm{CZF}_{\mathrm{f}}$}

The axiom system CZF for constructive set theory has usually been axiomatised in the first order language with equality and one binary infix relation symbol $\in$. Here we give an axiomatisation in our free logic, where we allow the formation of comprehension terms ${ }^{2}\{x \mid \phi\}$ for arbitrary formulae $\phi$ and have the following general non-logical axioms and schemes. Further axioms and schemes will be given after we have introduced some abbreviations. In the following $\phi$ is any formula and $a, b$ are terms.

Comprehension: $\quad a \in\{x \mid \phi\} \leftrightarrow a \downarrow \wedge \phi[a / x]$.

Extensionality: $\quad(\forall x)(x \in a \leftrightarrow x \in b) \rightarrow a=b$.

Elements are Sets: $\quad(a \in b) \rightarrow a \downarrow$.

\section{Some Abbreviations}

In these abbreviations $a, a_{1}, a_{2}, b, c, r$ are terms; i.e. either variables or comprehension terms. There may be a standard constraint that a variable is not allowed to occur free in a formula or term. For example in the abbreviation for $\{x \in a \mid \phi\}$ the variable $x$ should not occur free in the term $a$ and in the abbreviation for $(\exists ! x) \phi$ the variable $y$ should not occur free in $\phi$.

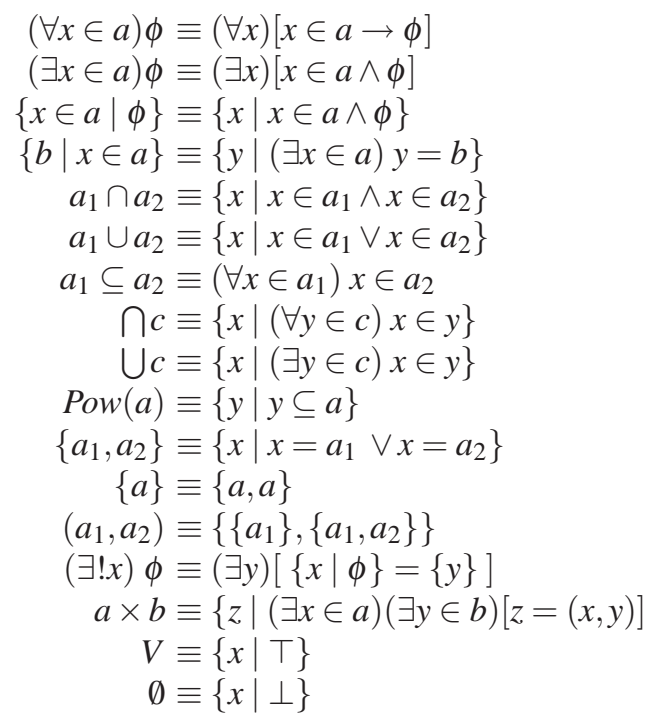

\footnotetext{
${ }^{2}$ Free occurrences of $x$ in $\phi$ become bound in $\{x \mid \phi\}$.
} 


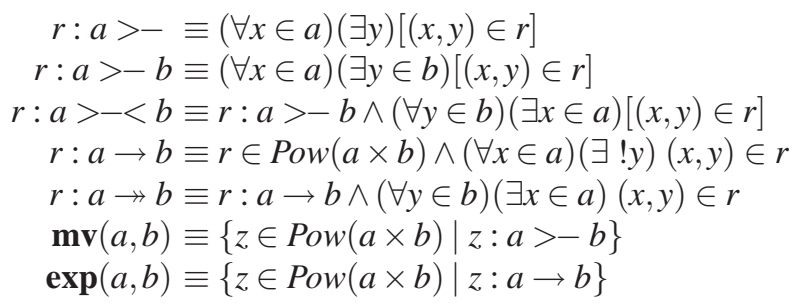

For our set theoretic representation of the natural numbers we use the following abbreviations.

$$
\begin{aligned}
0 & \equiv \emptyset \\
a^{+} & \equiv a \cup\{a\} \\
I N D_{\omega}(a) & \equiv 0 \in a \wedge(\forall y \in a)\left[y^{+} \in a\right] \\
\mathbb{N} & \equiv \bigcap\left\{x \mid I N D_{\omega}(x)\right\}
\end{aligned}
$$

Set Induction Scheme: For each term $a$,

$$
\operatorname{Pow}(a) \subseteq a \rightarrow V \subseteq a .
$$

Mathematical Induction Scheme: For each term $a$,

$$
I N D_{\omega}(a) \rightarrow \mathbb{N} \subseteq a .
$$

\section{The Set Existence Axioms and Schemes}

Pairing: $\quad\left(\forall x_{1}, x_{2}\right)\left\{x_{1}, x_{2}\right\} \downarrow$.

Union: $(\forall z) \cup z \downarrow$.

Restricted Separation Scheme: $\quad(\forall y)\{x \in y \mid \theta\} \downarrow$,

for each restricted formula $\theta$; i.e. formula $\theta$ in which each quantifier occurs in one of the forms $(\forall u \in v)$ or $(\exists u \in v)$.

Strong Infinity: $\mathbb{N} \downarrow$.

Strong Collection Scheme: $\quad(\forall x)[r: x>-\rightarrow(\exists y) r: x>-<y]$, for each term $r$.

Fullness: $\quad(\forall x)(\forall y)(\exists z \in \operatorname{Pow}(\mathbf{m v}(x, y)))(\forall u \in \mathbf{m v}(x, y))\left(\exists u_{0} \in z\right)\left[u_{0} \subseteq u\right]$.

\section{Remarks}

1. Using the Strong Collection Scheme both the Mathematical Induction Scheme and the Strong Infinity axiom can be derived using the following axiom.

Infinity: $\quad(\exists x) I N D_{\omega}(x)$.

2. The original formulation of $\mathbf{C Z F}$ used a certain scheme, the Subset Collection Scheme, instead of the Fullness axiom. That scheme and the Fullness axiom are equivalent, given the other axioms and schemes. 
3. Easy consequences of the Strong Collection Scheme and the Fullness axiom are the following scheme and axiom, respectively.

Replacement Scheme: $\quad a \downarrow \wedge(\forall x \in a)(\exists ! y) \phi \rightarrow\{y \mid(\exists x \in a) \phi\} \downarrow$, for each term $a$.

Exponentiation: $\quad a \downarrow \wedge b \downarrow \rightarrow \exp (a, b) \downarrow$, for arbitrary terms $a, b$.

We call this free formulation of $\mathbf{C Z F}, \mathbf{C Z F}_{\mathbf{f}}$.

Theorem: 3.1 $\mathbf{C Z F}_{\mathrm{f}}$ is a conservative extension of $\mathbf{C Z F}$.

Proof. We only sketch the idea for the straightforward proof. We can define a translation of the language of $\mathbf{C Z F}_{\mathbf{f}}$ into that of $\mathbf{C Z F}$ by systematically eliminating comprehension terms. This can be done by repeatedly replacing each equality $(a=b)$ by $(\forall x)[x \in a \leftrightarrow x \in b]$, each formula $(a \in b)$, where $a$ is a comprehension term by $(\exists x)[(\forall y)(y \in a \leftrightarrow y \in x) \wedge x \in b]$ and each formula $(x \in\{y \mid \phi\})$ by $\phi[x / y]$. Eventually all comprehension terms will be eliminated from a formula $\phi$ giving a formula $\phi^{\#}$ such that $\phi \leftrightarrow \phi^{\#}$ is provable in $\mathbf{C Z F}_{\mathbf{f}}$ and if $\phi$ is provable in $\mathbf{C Z F}_{\mathbf{f}}$ then $\phi^{\#}$ is provable in CZF. If $\phi$ already is in the language of $\mathbf{C Z F}$ then $\phi^{\#} \equiv \phi$ so that if it is provable in $\mathbf{C Z F}_{\mathbf{f}}$ then it is provable in $\mathbf{C Z F}$.

\subsection{The Axiom System $\mathrm{CZF}_{\mathrm{f}} \mathrm{I}$}

By leaving out the Set Induction Scheme from $\mathbf{C Z F}_{\mathbf{f}}$ we get the free version $\mathbf{C Z F}_{\mathbf{f}}{ }^{-}$ of $\mathbf{C Z F}^{-}$. We can also get the free version $\mathbf{C Z F}_{\mathbf{f}} \mathbf{I}$ of $\mathbf{C Z F I}$ by adding the binary relation symbol $\vdash$ to the language of $\mathbf{C Z F}_{\mathbf{f}}{ }^{-}$and adding the following axioms and scheme where, for terms $c, b$,

$$
\begin{aligned}
\operatorname{Closed}(c, b) & \equiv(\forall y \in \operatorname{Pow}(b))(\forall x)[(y, x) \in c \rightarrow x \in b], \\
I(c) & \equiv\{x \mid(\exists z \in \operatorname{Pow}(c)) z \vdash x\} .
\end{aligned}
$$

ind0: $\quad\left(\forall z, z^{\prime}\right)\left[z \subseteq z^{\prime} \rightarrow(\forall x)\left(z \vdash x \rightarrow z^{\prime} \vdash x\right)\right]$,

ind1: $\forall z \operatorname{Closed}(z, I(z))$,

ind2: $\forall z[\operatorname{Closed}(z, b) \rightarrow I(z) \subseteq b]$, for each term $b$.

\subsection{The Axiom System $\mathrm{CZF}_{\mathrm{f}}{ }^{*}$}

We formulate the free version of $\mathbf{C Z F}^{*}$. For each term $c$ let

$$
\begin{aligned}
I_{0}(c) & \equiv \bigcap\{y \mid \operatorname{Closed}(c, y)\} \\
I(c) & \equiv \bigcup\left\{I_{0}(z) \mid z \in \operatorname{Pow}(c)\right\}
\end{aligned}
$$

Note that $\operatorname{Closed}(I(c), c)$ is a theorem of $\mathbf{C Z F}_{\mathbf{f}}{ }^{-}$. 
Let

$\operatorname{Bounded}(c) \equiv \forall y\{x \mid(y, x) \in c\} \downarrow \wedge(\exists z)(\forall y \in \operatorname{dom}(c))(\exists v \in z)(\exists w) w: v \rightarrow y$,

where $\operatorname{dom}(c) \equiv\{y \mid \exists x(y, x) \in c\}$.

Bounded Induction Scheme (BIS): For terms $c, a$,

$$
[\operatorname{Closed}(c, a) \rightarrow I(c) \subseteq a] \wedge[\text { Bounded }(c) \rightarrow I(c) \downarrow]
$$

\section{Strong Set Compactness (SSC):}

$$
(\forall z)(\exists y \in \operatorname{Pow}(\operatorname{Pow}(z)))\left(\forall z^{\prime} \in \operatorname{Pow}(z)\right)\left[I_{0}\left(z^{\prime}\right)=\bigcup\left\{I_{0}\left(z_{0}\right) \mid z_{0} \in \operatorname{Pow}\left(z^{\prime}\right) \cap y\right\}\right]
$$

Finally we let $\mathbf{C Z F}_{\mathbf{f}}{ }^{*} \equiv \mathbf{C Z F}_{\mathbf{f}}^{-}+\mathbf{B I S}+\mathbf{S S C}$.

\section{Local Intuitionistic Zermelo Set Theory}

We outline a formal system LIZ which is a local version of Intuitionistic Zermelo set theory in which there is a one element sort $\mathbf{1}$, an infinite ground sort $\mathbf{N}$, product sorts $\alpha \times \beta$ and power sorts $\mathscr{P} \alpha$. The sorts have a natural interpretation in a set theory such as IZ, Intuitionistic Zermelo Set Theory, each sort $\alpha$ being interpreted as a set $[[\alpha]]$, with $[[\mathbf{1}]]=\{\emptyset\},[[\mathbf{N}]]$ any set with $\emptyset \in[[\mathbf{N}]]$ that is closed under $n \mapsto n \cup\{n\}$, and $[[\alpha \times \beta]]=[[\alpha]] \times[[\beta]]$, using the standard defintion of the cartesian product of two sets, and $[[\mathscr{P} \alpha]]=\operatorname{Pow}([[\alpha]]$, where Pow is the powerset operation on sets. With obvious interpretations of the constants $*, 0$ and function symbols ()$^{+},($,$) ,$ used below in forming terms, the axioms of LIZ, given below, are easily seen to be theorems of IZ.

There is an unlimited supply of variables of each sort. The terms of each sort and the formulae are simultaneously inductively generated. Here are the rules for generating terms.

1. Every variable of sort $\alpha$ is a term of sort $\alpha$.

2. $*$ is a term of sort $\mathbf{1}$.

3. 0 is a term of sort $\mathbf{N}$.

4. If $a$ is a term of sort $\mathbf{N}$ then $a^{+}$is also a term of sort $\mathbf{N}$.

5. If $a, b$ are terms of sorts $\alpha, \beta$ respectively then $(a, b)$ is a term of sort $\alpha \times \beta$.

6. If $\phi$ is a formula and $x$ is a variable of sort $\alpha$ then $\{x: \alpha \mid \phi\}$ is a term of sort $\mathscr{P} \alpha$.

$\mathrm{n}$ We call terms of the form $\{x: \alpha \mid \phi\} \alpha$-classes and write $a: \alpha$ when $a$ is an $\alpha$ term; i.e. a term of sort $\alpha$. The atomic formulae have one of the forms $\left(a_{1}=\alpha a_{2}\right)$ for $\alpha$-terms $a_{1}, a_{2}$ or $\left(a \in_{\alpha} b\right)$ when $a$ is an $\alpha$-term and $b$ is a $\mathscr{P} \alpha$-term. We will usually supress the subscript $\alpha$. 
Formulae are generated from the atomic formulae in the usual way using the logical constants $\perp$ and $T$, the binary connectives $\wedge, \vee$ and $\rightarrow$ and the quantifiers $(\forall x: \alpha)$ and $(\exists x: \alpha)$ for each variable $x$ of sort $\alpha$. Abbreviations for $\neg$ and $\leftrightarrow$ are defined as usual.

We use the following axiomatisation of Intuitionistic many sorted logic with equality. Any set of axioms and rules of inference for intuitionistic propositional logic will do. We just focus on the axioms and rules for equality and the quantifiers. The equality axioms are as follows, where $a, a_{1}, a_{2}$ are $\alpha$ terms and $x$ is a variable of sort $\alpha$.

$$
a=a \quad\left(a_{1}=a_{2}\right) \wedge \phi\left[a_{1} / x\right] \rightarrow \phi\left[a_{2} / x\right]
$$

We use the following quantifier rules and axioms, where $x$ is a variable of sort $\alpha$ not free in the formula $\theta$ and $a$ is an $\alpha$-term.

$$
\begin{array}{ll}
\frac{\theta \rightarrow \phi}{\theta \rightarrow(\forall x: \alpha) \phi} & (\forall x: \alpha) \phi \rightarrow \phi[a / x] \\
\phi[a / x] \rightarrow(\exists x: \alpha) \phi & \frac{\phi \rightarrow \theta}{(\exists x: \alpha) \phi \rightarrow \theta}
\end{array}
$$

We assume the following non-logical axioms and scheme, where $a_{1}, a_{2}, a$ are $\alpha$-terms, $b_{1}, b_{2}$ are $\beta$-terms, $c$ is a $\alpha \times \beta$-term and $d_{1}, d_{2}$ are $\mathscr{P} \alpha$-terms.

1. $\left(a_{1}, b_{1}\right)=\left(a_{2}, b_{2}\right) \rightarrow\left(a_{1}=a_{2}\right) \wedge\left(b_{1}=b_{2}\right)$

2. $(\exists x: \alpha)(\exists y: \beta)[c=(x, y)]$

3. $(\forall z: \mathbf{1})[z=*]$

4. $(\forall x: \alpha)\left(x \in d_{1} \leftrightarrow x \in d_{2}\right) \rightarrow d_{1}=d_{2}$

5. $(\forall y: \alpha)[y \in\{x: \alpha \mid \phi\} \leftrightarrow \phi[y / x]]$, for each formula $\phi$.

6. $(\forall x: \mathbf{N}) \neg\left(x^{+}=0\right)$

7. $\left(\forall x_{1}, x_{2}: \mathbf{N}\right)\left[\left(x_{1}^{+}=x_{2}^{+}\right) \rightarrow\left(x_{1}=x_{2}\right)\right]$

\section{Some Abbreviations}

It is convenient to introduce the following abbreviations where $a, a_{1}, a_{2}$ are $\mathscr{P} \alpha$ terms, $b$ is a $\beta$-term and $c$ is a $\mathscr{P} \mathscr{P} \alpha$-term. 


$$
\begin{aligned}
(\forall x \in a) \phi & \equiv(\forall x: \alpha)[x \in a \rightarrow \phi] \\
(\exists x \in a) \phi & \equiv(\exists x: \alpha)[x \in a \wedge \phi] \\
\{x \in a \mid \phi\} & \equiv\{x: \alpha \mid x \in a \wedge \phi\} \\
\{b \mid x \in a\} & \equiv\{y: \beta \mid(\exists x \in a) y=b\} \\
a_{1} \cap a_{2} & \equiv\left\{x: \alpha \mid x \in a_{1} \wedge x \in a_{2}\right\} \\
a_{1} \cup a_{2} & \equiv\left\{x: \alpha \mid x \in a_{1} \vee x \in a_{2}\right\} \\
a_{1} \subseteq a_{2} & \equiv\left(\forall x \in a_{1}\right) x \in a_{2} \\
\bigcap c & \equiv\{x: \alpha \mid(\forall y \in c) x \in y\} \\
\bigcup c & \equiv\{x: \alpha \mid(\exists y \in c) x \in y\} \\
P o w(a) & \equiv\{y: \mathscr{P} \alpha \mid y \subseteq a\} \\
(\exists ! x: \alpha) \phi & \equiv(\exists y: \alpha)[\{x \mid \phi\}=\{y\}] \\
a \times b & \equiv\{z: \alpha \times \beta \mid(\exists x \in a)(\exists y \in b)[z=(x, y)]
\end{aligned}
$$

As in the previous section we use the following abbreviations where now $a$ is a $\mathscr{P} \alpha$-term, $b$ is a $\mathscr{P} \beta$-term and $r$ is a $\mathscr{P}(\alpha \times \beta)$-term.

$$
\begin{aligned}
r: a>- & \equiv(\forall x \in a)(\exists y)[(x, y) \in r] \\
r: a>-b & \equiv(\forall x \in a)(\exists y \in b)[(x, y) \in r] \\
r: a>-<b & \equiv r: a>-b \wedge(\forall y \in b)(\exists x \in a)[(x, y) \in r] \\
r: a \rightarrow b & \equiv r \in \operatorname{Pow}(a \times b) \wedge(\forall x \in a)(\exists ! y)(x, y) \in r \\
r: a \rightarrow b & \equiv r: a \rightarrow b \wedge(\forall y \in b)(\exists x \in a)(x, y) \in r \\
\mathbf{m v}(a, b) & \equiv\{z \in \operatorname{Pow}(a \times b) \mid z: a>-b\} \\
\exp (a, b) & \equiv\{z \in \operatorname{Pow}(a \times b) \mid z: a \rightarrow b\}
\end{aligned}
$$

\section{The Natural Numbers}

The natural numbers are here represented using the following abbreviations where $c$ is a $\mathscr{P} \mathbf{N}$-term.

$$
\begin{aligned}
I_{N D_{\omega}(c)} & \equiv 0 \in c \wedge(\forall x \in c) x^{+} \in c \\
\mathbb{N} & \equiv \bigcap\left\{z: \mathscr{P N} \mid I_{N} D_{\omega}(z)\right\}
\end{aligned}
$$

We have not included any mathematical induction scheme for $\mathbf{N}$, as we can show that $\left(\mathbb{N}, 0,()^{+}\right)$satisfies the Dedekind-Peano axioms, including the mathematical induction axiom:

$$
(\forall z \in \operatorname{Pow}(\mathbb{N}))\left[\operatorname{IND}_{\omega}(z) \rightarrow \mathbb{N} \subseteq z\right]
$$

\subsection{Inductive Definitions in LIZ}

By an $\alpha$-inductive definition we simply mean a term $c: \mathscr{P}(\mathscr{P} \alpha \times \alpha)$ for some sort $\alpha$. If $b: \mathscr{P} \alpha$ let $I_{0}(c) \equiv \bigcap\{y: \mathscr{P} \alpha \mid \operatorname{Closed}(c, y)\}$, where

$$
\operatorname{Closed}(c, b) \equiv(\forall y \in \operatorname{Pow}(b))(\forall x: \alpha)[(y, x) \in c \rightarrow x \in b] \text {. }
$$


Because of the full impredicativity of LIZ the set $I_{0}(c)$ is provably the smallest $c$-closed set; i.e. the following sentences can be derived in LIZ for each term $c$ of sort $\mathscr{P}(\mathscr{P} \alpha \times \alpha)$.

$\operatorname{ind}_{0}$ 1 : $\quad(\forall z: \mathscr{P}(\mathscr{P} \alpha \times \alpha)) \operatorname{Closed}\left(z, I_{0}(z)\right)$,

ind $_{0}$ 2: $\quad(\forall z: \mathscr{P}(\mathscr{P} \alpha \times \alpha))(\forall y: \mathscr{P} \alpha)\left[\operatorname{Closed}(z, y) \rightarrow I_{0}(z) \subseteq y\right]$,

\section{Some Axiom Systems for Local CST}

The formal system LIZ is a thoroughly impredicative axiom system, each set having its powerset. We wish to have a predicative version of it, which will be a local version, $\mathbf{L C Z F}_{\mathbf{f}}{ }^{-}$, of $\mathbf{C Z F}_{\mathbf{f}}{ }^{-}$, to which we can add the forms of inductive definition of sets and classes that are available in constructive set theory, thereby giving us a generalised predicative formal system for local constructive set theory.

We keep the same terms and formulae of LIZ, but use a free version of the logic where the sorts are intended to be interpreted as classes of constructive set theory which are generally not accepted as sets. In particular the sort $\mathscr{P} \alpha$ is intended to be interpreted as the class of all subsets of the class interpreting the sort $\alpha$. So the comprehension terms of sort $\mathscr{P} \alpha$ can represent classes of values of sort $\alpha$ while not necessarily representing values in the range of the variables of sort $\mathscr{P} \alpha$, such variables only ranging over the sets of values of sort $\alpha$.

We modify the axiom system LIZ in the following way. For each $\alpha$-term $a$ let $a \downarrow$ abbreviate the formula $(\exists x: \alpha)(x=a)$, where the variable $x$ is chosen not to be free in the term $a$. The quantifier axioms are modified as follows.

$$
a \downarrow \wedge(\forall x: \alpha) \phi \rightarrow \phi[a / x] \quad a \downarrow \wedge \phi[a / x] \rightarrow(\exists x: \alpha) \phi
$$

In order to use these we need additional axioms that will enable us derive $a \downarrow$ for suitable terms $a$. In particular we need the axiom

$$
y \downarrow
$$

for each variable $y$. Also we need the following axioms for the sorts $\mathbf{1}, \mathbf{N}$ and $\alpha \times \beta$, where $a, b, c$ are terms of sorts $\alpha, \beta, \mathbf{N}$ respectively.

$$
\begin{aligned}
* \downarrow & & a \downarrow & \wedge b \downarrow \\
0 \downarrow & & c \downarrow & \leftrightarrow c^{+} \downarrow
\end{aligned}
$$

We also need, for each sort $\alpha$, the axioms $(a \in b) \rightarrow a \downarrow$, for terms $a, b$ of sorts $\alpha, \mathscr{P} \alpha$ respectively. 


\subsection{The Axiom System $\mathrm{LCZF}_{\mathrm{f}}^{-}$}

Mathematical Induction Scheme: $\quad I N D_{\omega}(a) \rightarrow(\mathbb{N} \subseteq a)$, for each $\mathbf{N}$-class $a$.

\section{Set Existence Axioms}

It remains to consider when we want $a \downarrow$ for $\mathscr{P} \alpha$-terms $a \equiv\{x: \alpha \mid \phi\}$. For this we have set existence axioms based on those for $\mathbf{C Z F}$.

Pairing: $\quad\left(\forall x_{1}, x_{2}: \alpha\right)\left\{x_{1}, x_{2}\right\} \downarrow$.

Union: $\quad(\forall z: \mathscr{P} \mathscr{P} \alpha) \cup z \downarrow$.

Restricted Separation Scheme: $\quad(\forall y: \mathscr{P} \alpha)\{x \in y \mid \theta\} \downarrow$,

for each restricted formula $\theta$; i.e. formula $\theta$ in which each quantifier occurs in one of the forms $(\forall u \in v)$ or $(\exists u \in v)$, where $v$ is a variable.

Strong Infinity: $\mathbb{N} \downarrow$.

Strong Collection Scheme: $\quad(\forall x: \mathscr{P} \alpha)[r: x>-\rightarrow(\exists y: \mathscr{P} \beta) r: x>-<y]$, for each $(\alpha \times \beta)$-class $r$.

\section{Fullness:}

$$
(\forall x: \mathscr{P} \alpha)(\forall y: \mathscr{P} \beta)(\exists z \in \operatorname{Pow}(\mathbf{m v}(x, y)))(\forall u \in \mathbf{m v}(x, y))\left(\exists u_{0} \in z\right)\left[u_{0} \subseteq u\right]
$$

We have now described our local version, $\mathbf{L C Z F}_{\mathbf{f}}{ }^{-}$, of $\mathbf{C} \mathbf{Z F}_{\mathbf{f}}{ }^{-}$.

\subsection{The Axiom System $\mathrm{LCZF}_{\mathrm{f}} \mathrm{I}$}

In order to have class inductive definitions in LCST we formulate a local version, $\mathbf{L} \mathbf{C Z F} \mathbf{f} \mathbf{I}$, of $\mathbf{C Z F}_{\mathbf{f}} \mathbf{I}$ by adding to the language of $\mathbf{L C Z F}_{\mathbf{f}}^{-}$a binary infix relation symbol $\vdash_{\alpha}$, for each sort $\alpha$. This relation symbol takes a first argument of sort $\mathscr{P}(\mathscr{P} \alpha \times \alpha)$ and a second argument of sort $\alpha$. We use the abbreviation for Closed $(c, b)$ as in the previous section. We add the following axioms and scheme, where for each term $c: \mathscr{P}(\mathscr{P} \alpha \times \alpha)$,

$$
\begin{aligned}
I_{o}(c) & \equiv\{x: \alpha \mid c \vdash x\}, \\
I(c) & \equiv \bigcup\left\{I_{0}(z) \mid z \in \operatorname{Pow}(c)\right\} .
\end{aligned}
$$

ind0 : $\quad\left(\forall z, z^{\prime}: \mathscr{P} \alpha\right)\left[z \subseteq z^{\prime} \rightarrow(\forall x: \alpha)\left(z \vdash_{\alpha} x \rightarrow z^{\prime} \vdash_{\alpha} x\right)\right]$,

ind1 : $\quad(\forall z: \mathscr{P}(\mathscr{P} \alpha \times \alpha)) \operatorname{Closed}(z, I(z))$,

ind2: $(\forall z: \mathscr{P}(\mathscr{P} \alpha \times \alpha))[\operatorname{Closed}(z, b) \rightarrow I(z) \subseteq b]$, for each term $b$. 


\subsection{The Axiom System $\mathrm{LCZF}_{\mathrm{f}}{ }^{*}$}

For each term $c: \mathscr{P}(\mathscr{P} \alpha \times \alpha)$, let

$$
\begin{aligned}
\operatorname{Bounded}(c) \equiv & (\forall y: \mathscr{P} \alpha)\{x: \alpha \mid(y, x) \in c\} \downarrow \\
& \wedge(\exists z: \mathscr{P} \mathscr{P} \beta)(\forall y \in \operatorname{dom}(c))(\exists v \in z)(\exists w: \mathscr{P}(\beta \times \alpha)) w: v \rightarrow y,
\end{aligned}
$$

where $\operatorname{dom}(c) \equiv\{y: \beta \mid(\exists x: \alpha)(y, x) \in c\}$.

Bounded Induction Scheme (BIS): For terms $c: \mathscr{P}(\mathscr{P} \alpha \times \alpha)$ and $a: \mathscr{P} \alpha$,

$$
[\text { Closed }(c) \rightarrow I(c) \subseteq a] \wedge[\text { Bounded }(c) \rightarrow I(c) \downarrow]
$$

Strong Set Compactness (SSC):

$$
\begin{aligned}
&(\forall z: \mathscr{P}(\mathscr{P} \alpha \times \alpha))(\exists y \in \operatorname{Pow}(\operatorname{Pow}(z))) \\
& \quad\left(\forall z^{\prime} \in \operatorname{Pow}(z)\right)\left[I_{0}\left(z^{\prime}\right)=\bigcup\left\{I_{0}\left(z_{0}\right) \mid z_{0} \in \operatorname{Pow}\left(z^{\prime}\right) \cap y\right\}\right]
\end{aligned}
$$

We let $\mathbf{L C Z F}_{\mathbf{f}}{ }^{*} \equiv \mathbf{L} \mathbf{C Z F} \mathbf{F}^{-}+\mathbf{B I S}+\mathbf{S S C}$.

\section{Well-founded Trees in Local CST}

In constructive mathematics the inductive definitions used to generate well-founded trees are particularly important. In the context of Martin-Löf's constructive type theory Martin-Löf introduced the inductively defined $W$-types $(W x: A) B(x)$, of wellfounded trees, where $B$ is a family of types indexed by a type $A$. The set theoretic version of $W$-types is naturally given as follows.

If $A, B$ are classes such that $B(x)=\{y \mid(x, y) \in B\}$ is a set for each $x \in A$ then we inductively define a class of well-founded trees, where at each node, the branching of the tree is indexed by one of the sets $B(a)$, for $a \in A$. So if $a \in A$ and $p(y)$ is a tree in the class for each $y \in B(a)$ then a tree $\sup (a, p)$ should be in the class having, as immediate subtrees, the trees $p(y)$, for $y \in B(a)$. In global set theory it is natural to represent $\sup (a, p)$ as simply the ordered pair $(a, p)$. This leads to the inductive definition of $W_{x \in A} B(x)$ as the smallest class $W$ such that $\Gamma W \subseteq W$ where, for each class $X$,

$$
\Gamma X=\sum_{x \in A} X^{B(x)}=\{(x, p) \mid x \in A \& p: B(x) \rightarrow X\} .
$$

So $W_{x \in A} B(x)=I(\Phi)$ where $\Phi$ is the inductive definition having the steps

$$
\operatorname{ran}(p) /(a, p)
$$

for $(a, p) \in \Gamma V$. 
This approach to representing $W$-types in CST is global. We would like to have a local version. We first need a suitable way to represent finite sequences in local CST. The following lemma gives us what we need.

Lemma: 6.1 For each class $X$ there is a class $\widehat{X}$, an element $<>\in \widehat{X}$ and an injective function $-:-: X \times \widehat{X} \rightarrow(\widehat{X}-\{<\rangle\})$.

Proof. It is enough to let $\widehat{X}=\operatorname{Pow}(\mathbb{N} \times X),<>=\emptyset$ and, for $a \in X$ and $\sigma \in \widehat{X}$,

$$
a: \sigma=\{(0, a)\} \cup\left\{\left(n^{+}, x\right) \mid(n, x) \in \sigma\right\} .
$$

Note that a finite sequence $a_{0}, a_{1}, \ldots, a_{n-1}$ of elements of $X$ is represented as the element of $\widehat{X}$

$$
a_{0}:\left(a_{1}:\left(\cdots\left(a_{n-1}:<>\right) \cdots\right)\right)
$$

which is just the set $\left\{\left(0, a_{0}\right),\left(1, a_{1}\right), \ldots,\left(n-1, a_{n-1}\right)\right\}$.

We will get a local version to the above global approach to representing $W$-types when we assume that each set $B(x)$ is a subset of some class $B_{0}$ and use $S: \Gamma C \rightarrow C$ given by the following result of local CST to represent the sup operation.

Theorem: $6.2\left(\mathbf{L C Z F}_{\mathbf{f}} \mathbf{I}\right)$ There is a class $C$ and an injective class function $S$ : $\Gamma C \rightarrow C$.

Proof. We define $C=\operatorname{Pow}(D)$ where $D=A \times \widehat{B_{0} \times A}$. For $d=(a, \sigma) \in D$ and $b \in B_{0}$ let

$$
b * d=((b, a): \sigma) \in \widehat{B_{0} \times A} .
$$

For $(a, p) \in \Gamma C$ let

$$
S(a, p)=\{a\} \times\left(\{<>\} \cup \bigcup_{b \in B(a)} p^{*}(b)\right) \in C
$$

where, for $b \in B(a)$,

$$
p^{*}(b)=\{b * d \mid d \in p(b)\} \in \operatorname{Pow}\left(\widehat{B_{0} \times A}\right) .
$$

To show that $S: \Gamma C \rightarrow C$ is injective, let $\left(a_{1}, p_{1}\right),\left(a_{2}, p_{2}\right) \in \Gamma C$ such that $S\left(a_{1}, p_{1}\right)=$ $S\left(a_{2}, p_{2}\right)$. Then, for $i=1,2$ and $x \in A$,

$$
x=a_{i} \Longleftrightarrow(x,<>) \in S\left(a_{i}, p_{i}\right)
$$

so that $a_{1}=a_{2}$. Let $a=a_{1}=a_{2}$ and $b \in B(a)$.

Claim: $p_{1}(b)=p_{2}(b)$.

Proof. By symmetry it suffices to show that every elementn of $p_{1}(b)$ is an element of $p_{2}(b)$. So let $d_{1}=\left(a_{1}^{\prime}, \sigma_{1}\right) \in p_{1}(b)$. Then $\left(\left(b, a_{1}^{\prime}\right): \sigma_{1}\right)=b * d_{1} \in p_{1}^{*}(b)$ so that $\left(a,\left(\left(b, a_{1}^{\prime}\right): \sigma_{1}\right)\right) \in$ $S\left(a, \sigma_{1}\right)=S\left(a, \sigma_{2}\right)$ and hence 


$$
\left(a,\left(\left(b, a_{1}^{\prime}\right): \sigma_{1}\right)\right)=\left(a, b_{2} * d_{2}\right)
$$

for some $b_{2} \in B(a)$ and some $d_{2}=\left(a_{2}^{\prime}, \sigma_{2}\right) \in p_{2}\left(b_{2}\right)$. So

$$
\left(\left(b, a_{1}^{\prime}\right): \sigma_{1}\right)=b_{2} * d_{2}=\left(\left(b_{2}, a_{2}^{\prime}\right): \sigma_{2}\right)
$$

so that $b=b_{2}, a_{1}^{\prime}=a_{2}^{\prime}$ and $\sigma_{1}=\sigma_{2}$ and hence

$$
d_{1}=\left(a_{1}^{\prime}, \sigma_{1}\right)=\left(a_{2}^{\prime}, \sigma_{2}\right)=d_{2} \in p_{2}\left(b_{2}\right)=p_{2}(b) ;
$$

i.e. $d_{1} \in p_{2}(b)$.

By the claim $p_{1}(b)=p_{2}(b)$ for all $b \in B(a)$ so that $p_{1}=p_{2}$ and hence $\left(a_{1}, p_{1}\right)=\left(a_{2}, p_{2}\right)$, proving the theorem.

Using $S$ let $W_{x \in A}^{\prime} B(x)=I\left(\Phi^{\prime}\right)$ where $\Phi^{\prime}$ is the inductive definition having the steps

$$
\operatorname{ran}(p) / S(a, p)
$$

for $(a, p) \in \Gamma C$. In order to show that this definition gives an adequate representation of $W$-types we need to show that the set theoretic version of the elimination rule for $W$-types can be proved; i.e. we need the following result, where $W=W_{x \in A}^{\prime} B(x)$.

Theorem: $6.3(\mathbf{L C Z F} \mathbf{f})$ If $C^{\prime}$ is a class and $S^{\prime}: \Gamma C^{\prime} \rightarrow C^{\prime}$ then there is a unique class function $K: W \rightarrow C^{\prime}$ such that, for $(a, p) \in \Gamma W$,

$$
K(S(a, p))=S^{\prime}(a, K \circ p) .
$$

Proof. We inductively define $K$ to be the smallest class such that if $(a, p) \in \Gamma C$ and $(a, q) \in \Gamma C^{\prime}$ such that

$$
\{(p(b), q(b)) \mid b \in B(a)\} \subseteq K
$$

then $\left(S(a, p), S^{\prime}(a, q)\right) \in K$. The theorem is a consequence of the following claims.

Claim 1: If $(w, z) \in K$ then $w \in W$.

Claim 2: If $w \in W$ then there is a unique $z \in C^{\prime}$ such that $(w, z) \in K$. By the previous claims $K: W \rightarrow C^{\prime}$.

Claim 3: If $(a, p) \in \Gamma W$ then $K(S(a, p))=S^{\prime}(a, K \circ p)$.

Claim 4: If $K^{\prime}: W \rightarrow C^{\prime}$ such that $K^{\prime}(S(a, p))=S^{\prime}\left(a, K^{\prime} \circ p\right)$ for all $(a, p) \in \Gamma W$ then $K^{\prime}=K$.

Claim 1 is proved by induction following the inductive definition of $K$ while Claims 2 and 4 are proved by induction following the inductive definition of $W$.

Theorem: $6.4\left(\mathbf{L C Z F}_{\mathbf{f}}{ }^{*}\right)$ If $A$ is a set then so is $W$.

Proof. Assume that $A$ is a set and observe that the inductive definition $\Phi^{\prime}$ of $W$ is bounded with set bound $\{B(a) \mid a \in A\}$. This is because, for each set $X$, if $X / S(a, p)$ is a step of $\Phi^{\prime}$ then $p$ maps $B(a)$ onto $X$, and the class of all $S(a, p)$ such that $X / S(a, p)$ is a step of $\Phi^{\prime}$ is the class

$$
\bigcup_{b \in B(a)}\{S(a, p) \mid p: B(a) \rightarrow X\},
$$


which is a set using Exponentiation, Replacement and Union. So, using the Bounded Induction Scheme we see that $W$ is a set.

\section{References}

1. Peter Aczel, Aspects of General Topology in Constructive Set Theory

2. Peter Aczel and Michael Rathjen, Notes on Constructive Set Theory, Institut Mittag-Leffler, Report No. 40, 2000/2001.

3. Peter Aczel and Michael Rathjen, Notes on Constructive Set Theory, available at http://www.mims.manchester.ac.uk/logic/mathlogaps/ workshop/CST-book-June-08.pdf, Mathlogaps workshop, Manchester, 2008.

4. Michael J. Beeson. Foundations of Constructive Analysis, Springer-Verlag, 1985.

5. John L. Bell. Toposes and Local Set Theories; An Introduction, Oxford Logic Guides, Clarendon Press, Oxford, 1988. 